\title{
Chlorhexidine hexametaphosphate as a wound care material coating: antimicrobial efficacy, toxicity and effect on healing
}

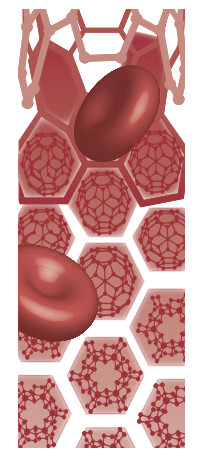

\begin{abstract}
Aim: In this study, chlorhexidine hexametaphosphate (CHX-HMP) is investigated as a persistent antimicrobial coating for wound care materials. Materials \& methods: $\mathrm{CHX}$ HMP was used as a wound care material coating and compared with chlorhexidine digluconate materials with respect to antimicrobial efficacy, toxicity and wound closure. Results: Antimicrobial efficacy at day 1, 3 and 7 was observed with experimental and commercial materials. CHX-HMP coated materials had less toxic effect on human placental cells than commercial chlorhexidine dressings. CHX-HMP in pluronic gel did not delay healing but reduced wound colonization by $E$. faecalis. Conclusion: $\mathrm{CHX}-$ HMP could become a useful component of wound care materials with sustained antimicrobial efficacy, lower toxicity than chlorhexidine digluconate materials, and reduction in wound colonization without affecting closure.
\end{abstract}

First draft submitted: 2 March 2016; Accepted for publication: 17 May 2016; Published online: 28 July 2016

Keywords: antimicrobial $\bullet$ catheter $\bullet$ chlorhexidine $\bullet$ healing $\bullet$ safety $\bullet$ toxicity $\bullet$ wound care

Venous catheters facilitate access to the circulatory system for monitoring, drug delivery and nutritional support, but are at risk of colonization by pathogenic bacteria. Infection is initially local, but may become systemic via the bloodstream, causing a catheter-related bloodstream infection (CRBSI). Incidence varies according to the condition that requires the use of a venous catheter and many other factors, but it has been reported that between 7 and $26 \%$ of venous catheter users with a range of different underlying conditions develop CRBSI [1-4]. CRBSI has many adverse clinical consequences; hospital stay is likely to be extended and there is a small but significant increase in absolute mortality of intensive care patients of $1 \%$ [5]. There is also, of course, a financial cost: CRBSI carries a cost of \$6-60k per event and often results in the premature removal of the catheter [6-8].

Wound care materials are used at the entry site of the catheter to hold the catheter in position and, potentially, to protect against infection. Catheter entry site dressings are traditionally either dry gauze and tape, or polyurethane-based materials [9]. The latter offer benefits such as clear visibility of the catheter entry site, improved durability and adhesive properties. Concerns have been raised, however, that polyurethane-based dressings maintain a high level of humidity at the entry site, and that this might contribute to a greater risk of colonization by microorganisms, and thus CRBSI [9]. Chlorhexidine (CHX)-impregnated dressings may be used to reduce this risk. $\mathrm{CHX}$, most commonly used as the soluble salt CHX digluconate (CHXdg), is a broad spectrum biocide, efficacious against a wide range of Gram-negative and -positive bacteria, including those implicated in CRBSI.

The aim of the study described here is the development of a prototype catheter dressing that incorporates particles of chlorhexidine hexametaphosphate (CHX-HMP). CHX-
Michele E Barbour*,1, Sarah E Maddocks², Helena J Grady ${ }^{1,3}$, James A Roper ${ }^{4}$, Mark D Bass $^{5}$, Andrew M Collins ${ }^{3}$, Rachel M Dommett ${ }^{6}$ \& Margaret Saunders ${ }^{7}$

'Oral Nanoscience, School of Oral \& Dental Sciences, University of Bristol, UK ${ }^{2}$ School of Health Sciences, Cardiff Metropolitan University, UK ${ }^{3}$ Bristol Centre for Functional Nanomaterials, University of Bristol, UK ${ }^{4}$ School of Biochemistry, University of Bristol, UK

${ }^{5}$ Centre for Membrane Interactions \& Dynamics, Department of Biomedical Science, University of Sheffield, UK ${ }^{6}$ School of Clinical Sciences, University of Bristol, UK

${ }^{7}$ Bioengineering, Innovation \& Research Hub (BIRCH), University Hospitals Bristol, UK

*Author for correspondence:

Tel.: +44 1173424419

m.e.barbour@bristol.ac.uk 
HMP is a sparingly soluble salt of $\mathrm{CHX}$ which forms, as a nanoparticulate salt, on reaction in water. When applied as a coating or dopant for biomaterials, it dissolves slowly in aqueous solutions and can thus provide sustained release of soluble CHX [10-12]. Here, CHX-HMP is used as a coating for a catheter entry site dressing, and its ability to inhibit bacterial growth is assessed, as well as its toxicological potential compared with $\mathrm{CHXdg}$, and the effect of the material on wound closure and wound infection in mice. The aim of this study was to investigate whether these CHX-HMP represent a viable option as a functional component for antimicrobial wound care materials.

\section{Materials \& methods}

Preparation of functionalized wound dressings CHX-HMP was prepared by mixing $10 \mathrm{mM}$ aqueous solutions of CHXdg and sodium hexametaphosphate using autoclaved deionized water at room temperature and pressure under ambient conditions and rapid stirring. Characterization of this CHX-HMP nanoparticulate precipitate has been reported in a previous publication by our group [10].

The starting wound dressing materials for microbiology experiments were Tegaderm $+\mathrm{Pad}^{\mathrm{TM}}$ and Tegaderm CHGTM (both 3M PLC, Bracknell, UK). Tegaderm + Pad is an adhesive dressing with a pad of dry, woven material in the center that contacts the wound. Tegaderm $\mathrm{CHG}$ is a transparent adhesive dressing with a pad of a gel material that contains $2 \% \mathrm{CHXdg}$ which contacts the wound. For each of the dressings, the central wound-contacting material was separated from the adhesive surrounding panel and sectioned into $10 \times$ $10 \mathrm{~mm}$ portions for use in the experiments, and the adhesive mounting material was discarded.

Two dressings were assessed as supplied: Tegaderm CHG as a positive control (T-CHG) and Tegaderm + Pad as a negative control (T-CTRL). Two prototype dressings were prepared: Tegaderm + Pad which was immersed for $30 \mathrm{~s}$ in a stirred suspension of 5 $\mathrm{mM}$ CHX-HMP followed by a $10 \mathrm{~s}$ rinse in deionized water (P-CHX-HMP) and Tegaderm +Pad which was immersed for $30 \mathrm{~s}$ in a stirred solution of $25 \mu \mathrm{M}$ $\mathrm{CHXdg}$ followed by a $10 \mathrm{~s}$ rinse in deionized water (P-CHG). $25 \mu \mathrm{M}$ is the residual concentration of aqueous $\mathrm{CHX}$ that is found in the $5 \mathrm{mM} \mathrm{CHX-HMP} \mathrm{prep-}$ aration, thus the $\mathrm{P}-\mathrm{CHG}$ dressing has been exposed to the same soluble CHX as the P-CHX-HMP dressing but without the CHX bound in the solid CHX-HMP precipitate.

T-CHG absorbed water rapidly on contact with culture medium and was therefore unsuitable for use as a positive control in the toxicology studies. In this phase of the experimental work, an alternative commercial wound dressing containing $0.5 \% \mathrm{CHX}$ diacetate was used: Bactigras (BAC; Smith and Nephew, Hull, UK) and compared with T-CTRL and P-CHX-HMP.

\section{Antimicrobial efficacy}

Staphylococcus aureus (EMRSA-15 and methicillin sensitive S. aureus), Pseudomonas aeruginosa (ATCC9027 and PA14, a highly virulent, multidrug resistant wound isolate), Klebsiella pneumoniae (ATCC10031), Acinetobacter baumanii (121J6), Escherichia coli (NCTC10418) and Staphylococcus epidermidis were routinely cultured in nutrient broth (Sigma Aldrich) aerobically at $37^{\circ} \mathrm{C}$. Clostridium difficile was cultured in tryptone soya broth (Sigma Aldrich) under anaerobic conditions (DW Scientific anaerobic cabinet) at $37^{\circ} \mathrm{C}$.

Wound dressings T-CHG, T-CTRL, P-CHG and P-CHX-HMP were compared with regard to antimicrobial efficacy immediately after preparation, after 7 days aging by immersion, and after 3 days aging under flow conditions, to investigate the efficacy of the wound dressings several days after it had been applied to the wound. To age the dressings by immersion, they were immersed in simulated wound fluid (SWF; $50 \% \mathrm{v} / \mathrm{v}$ fetal calf serum in maximum recovery diluent; Sigma Aldrich) for 7 days at room temperature under aerobic conditions. To age the dressings under flow conditions, they were placed in a sterile glass tube and SWF flowed over them for 3 days at $0.74 \mathrm{ml} / \mathrm{min}$ (Watson Marlow 505U peristaltic pump).

Dressings were aseptically transferred into the well of a 24 well microtiter plate and submerged in $1 \mathrm{ml}$ SWF. Each well was inoculated with the appropriate microorganism at $1.5 \times 10^{8} \mathrm{cfu} / \mathrm{ml}$ and incubated at $37^{\circ} \mathrm{C}$ for $16 \mathrm{~h}$ under aerobic conditions. Dressings were removed from the wells using sterile forceps and transferred into sterile plastic bijoux (Fisher Scientific) containing $1 \mathrm{ml}$ sterile phosphate buffered saline (Sigma Aldrich) and vortexed for $60 \mathrm{~s}$ to dislodge bacteria. Serial dilutions from $10^{-1}$ to $10^{-12}$ were prepared in sterile phosphate buffered saline and plated onto nutrient agar. Bacteria were enumerated using the method of Miles et al. [13]. For $C$. difficile the experiments described above were undertaken using an anaerobic cabinet (DW Scientific) and enumeration used tryptone soya agar.

Swabs were processed to maximize the recovery of aerobic and facultatively anaerobic bacteria of the skin and enteric origin, as typically colonize wounds. Each swab was cultured on nutrient agar (for microscopy), Blood agar, MacConkeys agar, Baird Parker agar and Mannitol Salt agar (all sourced from Sigma Aldrich) aerobically at $37^{\circ} \mathrm{C}$ for $24 \mathrm{~h}$. Pure culture colonies were Gram stained, catalase testing was performed for Gram positive cocci and oxidase testing performed 
for Gram negative rods. Presumptive Streptococci spp. were further analyzed by Streptex (Remel; UK) testing; presumptive Staphylococci were further analyzed by Staphaurex test (Remel; UK).

\section{Toxicology assays}

Reagents were Dulbecco's modified Eagle medium/ nutrient F-12 Ham (DMEM-F12) (supplemented with $1 \%(\mathrm{v} / \mathrm{v}) \quad$ L-glutamine-penicillin-streptomycin (PSLG), 1\% (v/v) amphotericin B solution (Amp B), and $10 \%(\mathrm{v} / \mathrm{v})$ fetal bovine serum, phosphate buffered saline, $0.25 \%$ trypsin-EDTA solution (Sigma-Aldrich, UK). Wound dressings examined were T-CTRL, P-CHX-HMP and BAC.

The placental choriocarcinoma cell line BeWo b30 was obtained from Prof. Harry McArdle (Rowett Research Institute, Aberdeen) with permission from Dr. Alan Schwartz (Washington University, MO, USA) [14]. Cells were cultured in $5 \% \mathrm{CO}_{2}$ at $37^{\circ} \mathrm{C}$ in a humidified atmosphere in complete DMEM-F12 medium with supplements. At confluence, the cells were subcultured using $0.25 \%$ trypsin-EDTA solution.

BeWo b30 cells were seeded at $1.75 \times 10^{5}$ cells $/ \mathrm{ml}$ in 12 well cell culture plates in $1 \mathrm{ml}$ volumes (Corning Costar, Sigma-Aldrich, UK), and grown until approximately $70 \%$ confluent ( $72 \mathrm{~h}$ post-seeding). Cells were washed with $1 \mathrm{ml}$ fresh medium, and incubated at $37^{\circ} \mathrm{C}, 5 \% \mathrm{CO}_{2}$ in a humidified atmosphere for $1 \mathrm{~h}$ prior to treatment. Cells in $1 \mathrm{ml}$ fresh cell culture medium were exposed to T-CTRL, T-CHX-HMP and BAC with unexposed cells as a negative control and cells exposed to $30 \%$ ( $1 \mathrm{mM}) \mathrm{H}_{2} \mathrm{O}_{2}$ prepared in cell culture medium as a positive control. Cells were exposed for 2 and $24 \mathrm{~h}$ to all treatments for toxicity testing.

Cytotoxicity was measured by monitoring the release of lactate dehydrogenase $(\mathrm{LDH})$ in the cell culture medium from unexposed cells, the positive control cells or treated cells which had been exposed to CHX-HMP-NP functionalized specimens, using the CytoTox $96^{\circledR}$ nonradioactive cytotoxicity assay kit (Promega, Southampton, UK). The assay was performed as per the manufacturer's instructions.

Briefly, after 2 and $24 \mathrm{~h}$, cell culture supernatants were collected and $50 \mu \mathrm{l}$ aliquots were transferred to a flat bottom 96 well plate (Corning Costar, SigmaAldrich, UK). $50 \mu \mathrm{l}$ of reconstituted assay buffer was added to each well containing the specimens, and plates were incubated in the dark for $30 \mathrm{~min}$ at room temperature. $50 \mu \mathrm{l}$ of the 'stop solution' that forms part of the CytoTox 96 cytotoxicity assay kit was then added to each well, and the plates were read in a multiwell plate reader (SpectraMax M Series Multi-Mode Microplate Reader, Molecular Devices, CA, USA) at $492 \mathrm{~nm}$. Optical density (OD) readings from wells containing cells exposed to treatment were expressed as a percentage of the untreated negative control: $\%$ of control $=$ OD treated $/ O D$ untreated $\times 100$.

Data are presented as mean $\pm S D$ of three independent experiments. Two-way analysis of variance (ANOVA) followed by Tukey post hoc multiple comparisons test was performed (GraphPad Prism version 6.00 for Windows, GraphPad Software, CA, USA). $\mathrm{p}<0.05$ was considered significant.

\section{Wound healing in mice}

Animal experiments were performed in accordance with Home Office regulations described by the UK Animals (Scientific Procedures) Act 1986. Threemonth old C57BL/6J mice were anesthetized by isofluorane inhalation and two pairs of full-thickness $4 \mathrm{~mm}$ excisional punch wounds were made either side of the dorsal midline of the barbered back of each animal. $30 \%$ pluronic gel (Sigma) preparations containing no additive, $30 \%$ pluronic gel with $50 \mu \mathrm{M}$ EHT1864 (Sigma Aldrich) or $30 \%$ pluronic gel with $5 \mathrm{mM}$ CHX-HMP particles were prepared as a liquid at $4^{\circ} \mathrm{C}$. Appropriate pluronic gel preparations were administered directly to the wound by pipette immediately after wounding and allowed to solidify.

Wound size was recorded by photographing mice at the same time each day after wounding. At 2, 3 and 4 days post wounding, wounds were swabbed with Transwab Amies (MWE) for microbiological analysis.

\section{Results}

Antimicrobial efficacy

$\log _{10}$ colony forming units $(\mathrm{CFU} / \mathrm{ml})$ of the nine microbes when exposed for $24 \mathrm{~h}$ to T-CTRL, P-CHG, P-CHX-HMP and T-CHG dressings after three aging conditions are shown, with standard errors, in Table 1. All bacteria grew readily in contact with T-CTRL. P-CHG caused a small reduction in growth of some species, particularly the Staphylococci species and E. coli. P-CHX-HMP eliminated microbial growth of all but one microorganism; for $A$. baumanii P-CHXHMP resulted in $5 \times \log _{10}$ reduction in CFU. T-CHG eliminated growth of all bacteria tested. Aging of the dressings had no significant effect on the bactericidal efficacy of T-CHG or P-CHX-HMP.

\section{Toxicology assays}

$\mathrm{LDH}$ release from BeWo cells exposed to T-CTRL, P-CHX-HMP and BAC is shown in Figure 1. Cells exposed to T-CTRL and P-CHX-HMP showed no difference in $\mathrm{LDH}$ release after 2 and 24 h compared with the negative control (media). BAC resulted in an $\mathrm{LDH}$ release similar to the positive control $\left(\mathrm{H}_{2} \mathrm{O}_{2}\right)$ after 2 and $24 \mathrm{~h}$ exposure. 
Table 1. $\log _{10}$ colony forming units (CFUs) of nine microbes when exposed to four dressings after three aging conditions, with standard errors in parentheses.

\begin{tabular}{|c|c|c|c|c|c|}
\hline \multirow[t]{2}{*}{ Microbe } & \multirow[t]{2}{*}{ Aging } & \multicolumn{4}{|c|}{$\log _{10}$ CFU (SE) } \\
\hline & & T-CTRL & P-CHG & P-CHX-HMP & T-CHG \\
\hline \multirow[t]{3}{*}{ S. epidermidis } & None & $8.17(0.04)$ & $3.01(0.01)$ & 0 & 0 \\
\hline & 7 days' immersion & $8.27(0.03)$ & $3.06(0.02)$ & 0 & 0 \\
\hline & 3 days' flow & $8.29(0.03)$ & $3.12(0.01)$ & 0 & 0 \\
\hline \multirow[t]{3}{*}{ S. aureus (MSSA) } & None & $8.25(0.03)$ & $3.03(0.01)$ & 0 & 0 \\
\hline & 7 days' immersion & $8.31(0.01)$ & $3.11(0.00)$ & 0 & 0 \\
\hline & 3 days' flow & $8.26(0.01)$ & $3.19(0.01)$ & 0 & 0 \\
\hline \multirow[t]{3}{*}{ S. aureus (MRSA) } & None & $8.89(0.28)$ & $3.08(0.00)$ & 0 & 0 \\
\hline & 7 days' immersion & $8.66(0.24)$ & $3.21(0.04)$ & 0 & 0 \\
\hline & 3 days' flow & $8.71(0.22)$ & $3.32(0.08)$ & 0 & 0 \\
\hline \multirow[t]{3}{*}{ P. aeruginosa } & None & $8.86(0.02)$ & $7.74(0.01)$ & 0 & 0 \\
\hline & 7 days' immersion & $9.06(0.15)$ & $7.73(0.02)$ & 0 & 0 \\
\hline & 3 days' flow & $8.98(0.16)$ & $7.75(0.02)$ & 0 & 0 \\
\hline \multirow[t]{3}{*}{ P. aeruginosa (MDR) } & None & $8.78(0.03)$ & $7.65(0.01)$ & 0 & 0 \\
\hline & 7 days' immersion & $8.74(0.02)$ & $7.67(0.01)$ & 0 & 0 \\
\hline & 3 days' flow & $8.72(0.01)$ & $7.67(0.01)$ & 0 & 0 \\
\hline \multirow[t]{3}{*}{ K. pneumoniae } & None & $8.40(0.03)$ & $7.69(0.00)$ & 0 & 0 \\
\hline & 7 days' immersion & $8.59(0.04)$ & $7.53(0.08)$ & 0 & 0 \\
\hline & 3 days' flow & $8.64(0.04)$ & $7.51(0.01)$ & 0 & 0 \\
\hline \multirow[t]{3}{*}{ E. coli } & None & $8.42(0.18)$ & 0 & 0 & 0 \\
\hline & 7 days' immersion & $8.66(0.06)$ & $4.83(0.04)$ & 0 & 0 \\
\hline & 3 days' flow & $8.64(0.08)$ & $4.91(0.04)$ & 0 & 0 \\
\hline \multirow[t]{3}{*}{ C. difficile } & None & $6.23(0.01)$ & $4.68(0.04)$ & 0 & 0 \\
\hline & 7 days' immersion & $6.29(0.03)$ & $6.01(0.20)$ & 0 & 0 \\
\hline & 3 days' flow & $6.36(0.04)$ & $6.11(0.09)$ & 0 & 0 \\
\hline \multirow[t]{3}{*}{ A. baumanii } & None & $10.48(0.01)$ & $7.36(0.03)$ & $4.80(0.04)$ & 0 \\
\hline & 7 days' immersion & $10.56(0.03)$ & $7.47(0.02)$ & $5.04(0.12)$ & 0 \\
\hline & 3 days' flow & $10.62(0.03)$ & $7.48(0.01)$ & $5.11(0.05)$ & 0 \\
\hline
\end{tabular}

Wound healing in mice

Relative wound area as a function of time is shown in Figure 2, and photographs of the wounds on successive days are shown in Figure 3. The proportion of wounds testing positive for E. faecalis is shown in Figure 4. Application of pluronic gel containing the Rac1-inhibitor, EHT-1864, that inhibits signaling downstream of growth factors and extracellular matrix, retarded wound closure compared with pluronic gel alone, as previously observed [15]. Wounds treated with pluronic gel containing CHX-HMP were indistinguishable from wounds treated with pluronic gel alone.

Microbiological analysis of swabs of the wounds revealed that wounds treated with pluronic gel alone were contaminated with Enterococcus faecalis at days
3-5 post-wounding (Figure 3). However, application of CHX-HMP in the pluronic gels impaired colonization over that time period, supporting the in vitro assessment of wound dressing efficacy in which the growth of Gram positive bacteria was inhibited.

\section{Discussion}

It is well established that $\mathrm{CHX}$-impregnated catheter dressings reduce the incidence of CRBSI [16]. A metaanalysis of nine randomized clinical trials indicated that the use of CHX dressings reduced both catheter bacterial colonization and CRBSI [17]. Despite the higher cost of CHX dressings, it has been estimated that the clinical benefits would be such that adoption of CHX dressings for catheter care would result in a 


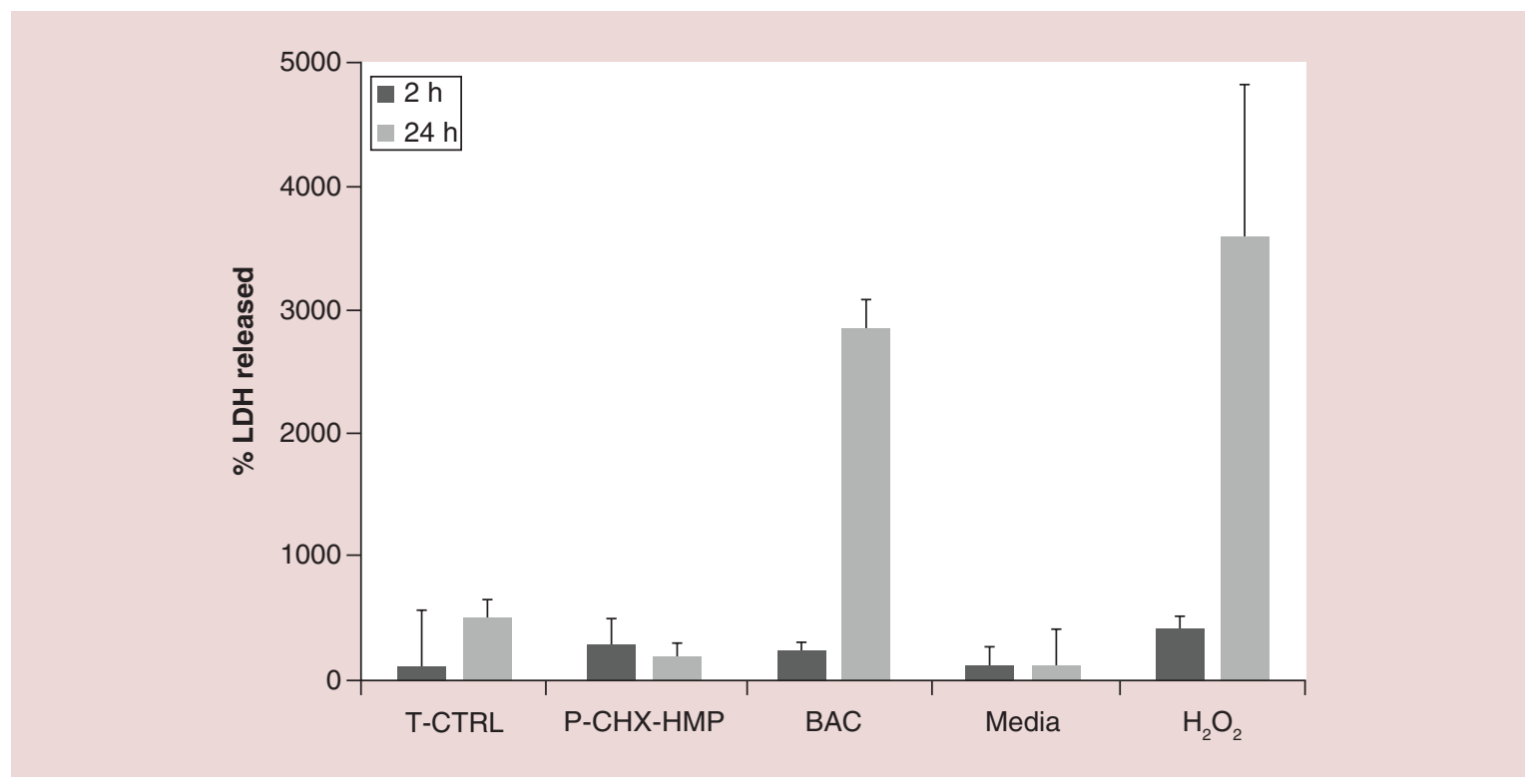

Figure 1. Lactate dehydrogenase (LDH) release from BeWo cells exposed to three wound dressings (T-CTRL, P-CHX-HMP and BAC), media (negative control) and $\mathrm{H}_{2} \mathrm{O}_{2}$ (positive control) for 2 and $24 \mathrm{~h}$.

saving of $£ 73$ per patient or $£ 4.2-10.8$ million per year in the UK [18]. Consequently, a CHX-impregnated dressing (Tegaderm CHG) was recommended for patients by the National Institute for Health and Clinical Excellence (NICE) in the UK in 2015 for high dependency patients and those in intensive care [19].

A number of other approaches to creating CHXcontaining dressings have been reported in the literature. There is particular interest in creating dressings which confer a sustained CHX-rich environment. CHX diacetate is often the preferred option in these experimental dressings, perhaps as it has a lower solubility than CHXdg and thus is selected on the basis that this might prolong the CHX release. CHX diacetate was incorporated into thermoresponsive polymer hydrogels and these offered a degree of sustained CHX release, although this persisted for only $2-24 \mathrm{~h}$ [20]. CHX diacetate was also incorporated into a multilayered polymeric dressing, creating a release profile that was sustained for at least 8 days and showing bacteriostatic and, at higher concentrations, bactericidal efficacy against $S$. aureus [21].

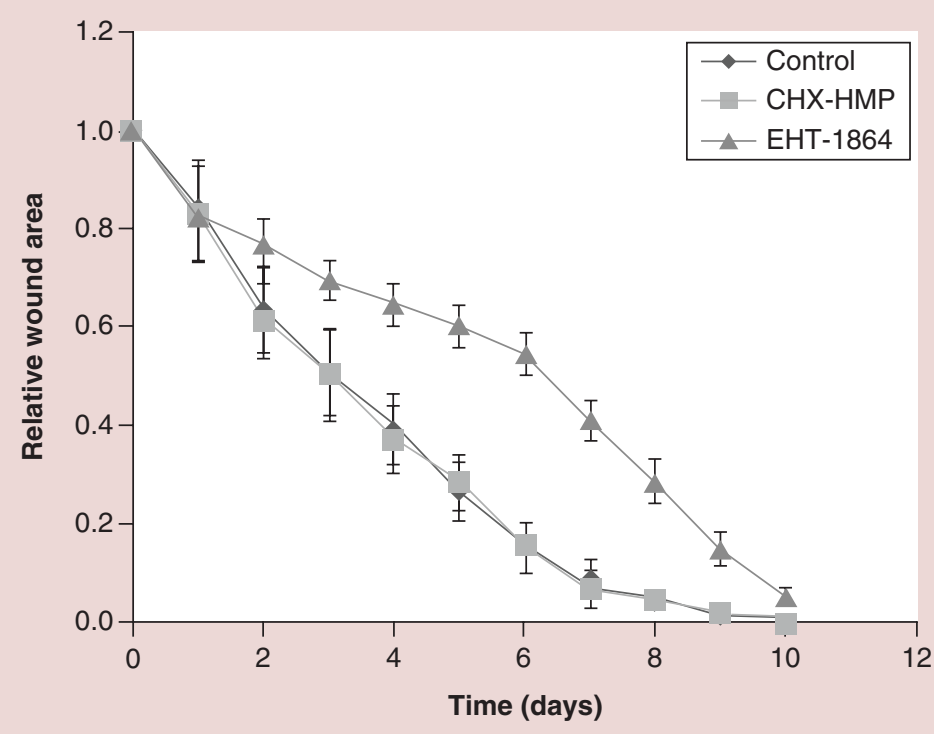

Figure 2. Relative wound area in mice treated with pluronic gel (control), pluronic gel with CHX-HMP and pluronic gel with the wound healing inhibitor EHT-1864. 


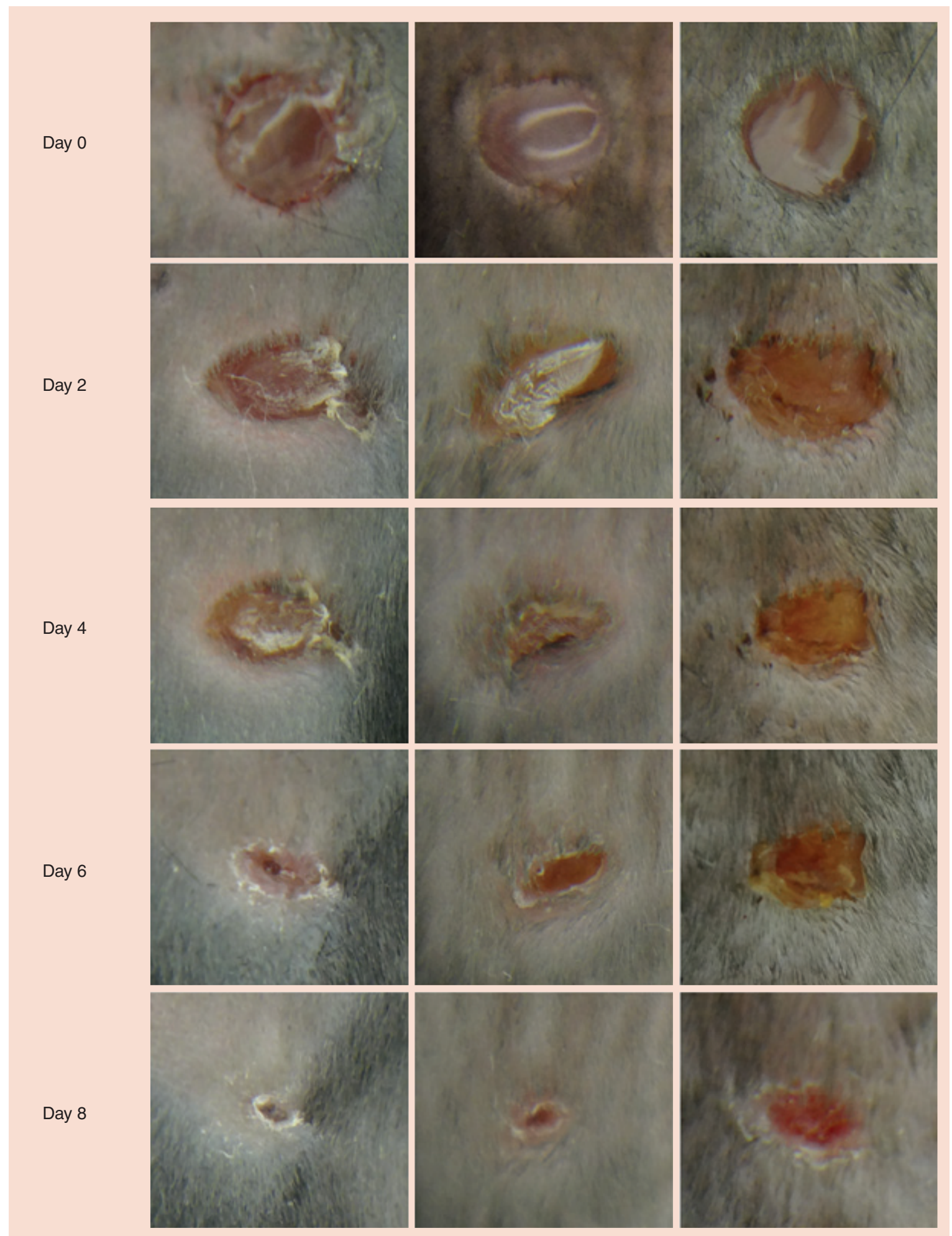

Figure 3. Photographs of wounds on mice on successive days. Column 1: control; column 2: CHX-HMP; column 3: EHT 1864, a wound healing inhibitor.

The approach described here utilized CHX-polyphosphate particles, specifically the hexametaphosphate salt of CHX. It is already known that these gradually dissolve in aqueous environments, providing a sustained release of soluble CHX, but they have not previously been applied for use in wound care. 


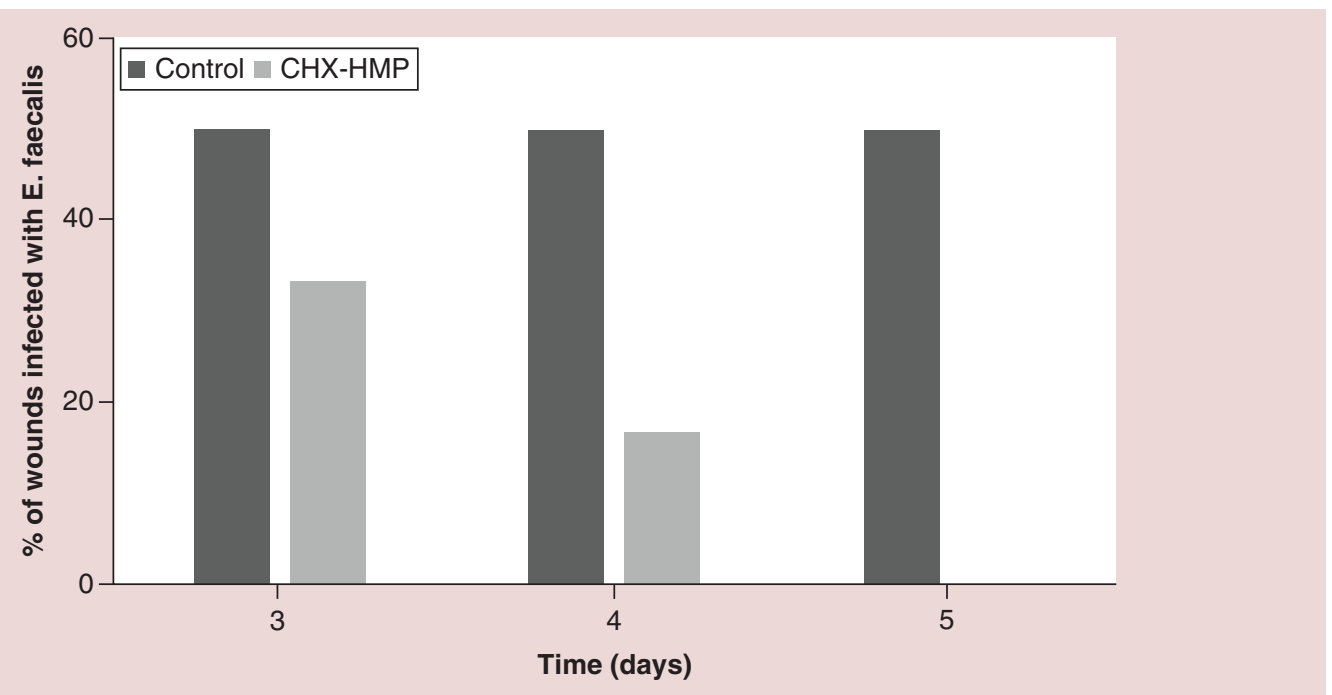

Figure 4. Proportion of mouse wounds infected with $E$. faecalis as a function of time and gel composition (control or CHX-HMP).

An equivalent antimicrobial efficacy to a commercial CHX-based catheter entry site dressing was demonstrated, with the exception of $A$. baumanii for which the commercial dressing was more efficacious. Both the commercial and experimental dressing exhibited bactericidal efficacy initially and after 3 or 7 days' ageing.

The cytotoxicity of CHXdg is well established, and is dose dependent. CHXdg exhibits cytotoxic effects against $\mathrm{HaCaT}$ keratinocytes at concentrations of $0.5 \%$ (by mass of the CHX ion, hence $1.0 \mathrm{mM}$ ) and above [22]. $0.2 \%(2.2 \mathrm{mM})$ chlorhexidine digluconate exhibited cytotoxic effects against human gingival fibroblasts and SaOS2-like cells [23]. It was not possible to use the Tegaderm CHG material as the control in this study, as the extremely hygroscopic material rapidly absorbed all of the culture medium; it was for this reason that a different commercial CHX containing dressing (Bactigras) was used which did not exhibit this behavior. The LDH release assay demonstrated that CHX-HMP coated wound dressings exhibited lower toxicity than the commercial CHX containing dressing; the CHX-HMP dressing was comparable to the negative control.

Healing rate could potentially be affected by both material toxicity and reduced microbial load in the wound. Inflammation is the initial phase of wound healing [24]. In addition to combating infection, infiltrating inflammatory cells secrete growth factors, extracellular matrix, reactive oxygen species and nitric oxide that drive the subsequent phases of wound contraction and re-epithelialization $[24,25]$, so that modification of the wound flora could have a knock-on effect on healing rates. Pluronic gel was used as a carrier medium for the CHX-HMP to examine the effect of CHX-HMP on wound healing rates; this was a convenient medium as it solidifies at room temperature, which prevents the mouse from removing the gel from the wound. Wounds treated with gel containing CHX-HMP were indistinguishable from those coated with unmodified gel in terms of healing time, but exhibited less $E$. faecalis, with this microbe eliminated at 5 days post wounding. Thus the CHX-HMP did not adversely affect wound healing, suggesting in these terms a biocompatible material, and it did reduce wound infection, which may provide benefit in terms of the development of pathological infection at catheter entry sites.

\section{Conclusion}

In conclusion, wound dressings coated with CHXHMP exhibited a similar antimicrobial profile to a commercial CHXdg containing wound care material for microbes MRSA, E. coli, K. pneumoniae and $P$. aeruginosa, but for $A$. baumanii the antimicrobial efficacy was less with the prototype material, although there was still a $5 \times \log 10$ reduction in colony forming units. Damage to human BeWo cells, as assessed by $\mathrm{LDH}$ release inferring membrane integrity, was not observed with CHX-HMP whereas a commercial CHX containing dressing elicited a similar response in the cells to the hydrogen peroxide positive control. Wound closure in mice was not adversely affected by CHX-HMP, but wound colonization by $E$. faecalis was reduced by CHX-HMP. Therefore we conclude that CHX-HMP is an effective antimicrobial agent in in vivo situations but does not have a negative effect on wound healing.

\section{Future perspective}

Multifunctional wound dressings are the subject of 
intensive research, and chief among these are antiinfective dressings. These functional biomaterials are of huge potential clinical impact, allowing physicians to prevent infection, and also treat infection which is already established. Nanoparticles offer much potential in this initiative, not least because their small size and high surface area can facilitate careful control of dose and duration of the antimicrobial agents used. In this age of burgeoning antimicrobial resistance, strategies which reduce or negate the need for antibiotics are likely to find increasing attention.

\section{Open access}

This article is distributed under the terms of the Creative Commons Attribution License 4.0 which permits any use, distribution, and reproduction in any medium, provided the original author(s) and the source are credited. To view a copy of the license, visit http://creativecommons.org/licenses/by/4.0/

\section{Data storage}

Supporting data are available from the University of Bristol research data repository at http://doi.org/10.5523/bris.5gmx 7o547hn419|4swdvc3azs

Financial \& competing interests disclosure One author (ME Barbour) is the named inventor of the chlorhexidine material in a patent application and is a founder and director of a University of Bristol spin-out company formed in 2015 to facilitate the commercial application of the technology. The research presented in this manuscript was funded by EPSRC under the Impact Acceleration Account scheme (EP-K503824). The authors have no other relevant affiliations or financial involvement with any organization or entity with a financial interest in or financial conflict with the subject matter or materials discussed in the manuscript apart from those disclosed.

No writing assistance was utilized in the production of this manuscript.

\section{Executive summary}

\section{Preparation of wound dressings}

- Chlorhexidine-hexametaphosphate (CHX-HMP) is a sparingly soluble salt of the widely used biocide chlorhexidine, and is formed rapidly as a nanoparticulate salt on combination of the aqueous solutions of the component ions.

- Commercial wound care materials were coated with CHX-HMP and compared with commercial materials containing chlorhexidine digluconate $(\mathrm{CHXdg})$ as well as unmodified dressings.

Antimicrobial efficacy

- Both the CHX-HMP and the CHXdg dressings exhibited good antimicrobial efficacy at 1, 3 and 7 days' aging.

- The only substantive difference in antimicrobial performance was with microbe Acenitobacter baumanii, for which the commercial CHXdg dressing eliminated bacterial growth but the CHX-HMP dressing, did not, and rather resulted in a $5 \times \log 10$ reduction in colony forming units.

Toxicology

- CHX-HMP coated dressings exhibited no deleterious effects toward human placental BeWo cells.

- A commercial $\mathrm{CHXdg}$ dressing did adversely affect the BeWo cells, giving a similar protein release to the positive control, hydrogen peroxide.

Wound healing in mice

- CHX-HMP applied in pluronic gel to mouse skin wounds had no impact on time to wound closure.

- Wounds treated with the CHX-HMP gel exhibited less colonization by E. faecalis.

Conclusion

- CHX-HMP provided effective antimicrobial control over at least 7 days, but did not adversely affect human placental cells, whereas a CHXdg dressing did.

- CHX-HMP did not adversely affect wound healing in mice, but did reduce colonization of the wound by E. faecalis.

\section{References}

Papers of special note have been highlighted as: $\bullet$ of interest; •• of considerable interest

1 Lorente L, Lecuona M, Jimenez A et al. Chlorhexidine-silver sulfadiazine- or rifampicin-miconazole-impregnated venous catheters decrease the risk of catheter-related bloodstream infection similarly. Am. J. Infect. Control 44(1), 50-53 (2016).

- This manuscript illustrates the clinical benefit of antimicrobial functionalized catheters.

2 Wang P, Wang Y, Qiao Y, Zhou S, Liang X, Liu Z. A retrospective study of preferable alternative route to right internal jugular vein for placing tunnelled dialysis catheters: right external jugular vein versus left internal jugular vein. PLoS ONE 11(1), e0146411 (2016).

3 Morano SG, Latagliata R, Girmenia C et al. Catheterassociated bloodstream infections and thrombotic risk in hematologic patients with peripherally inserted central catheters (PICC). Support. Care Cancer 23(11), 3289-3295 (2015).

4 Bech LF, Drustrup L, Nygaard L et al. Environmental risk factors for developing catheter-related bloodstream infection in home parenteral nutrition patients. A 6-year follow-up study. JPEN J. Parenter. Enteral. Nutr. pii: 0148607115579939 (2015) (Epub ahead of print). 
5 Halton KA, Cook D, Paterson DL, Safdar N, Graves N. Cost-effectiveness of a central venous catheter care bundle. PLoS ONE 5(9), pii: e12815 (2010).

-. This manuscript considers the economic aspects of catheter care bundles and antimicrobials, indicating that there is a net saving associated with reducing infection.

6 Cooper K, Frampton G, Harris P et al. Are educational interventions to prevent catheter-related bloodstream infections in intensive care unit cost-effective? J. Hosp. Infect. 86(1), 47-52 (2014).

7 Kim JS, Holtom P, Vigen C. Reduction of catheter-related bloodstream infections through the use of a central venous line bundle: epidemiologic and economic consequences. $\mathrm{Am}$. J. Infect. Control 39(8), 640-646 (2011).

8 Schwebel C, Lucet JC, Vesin A et al. Economic evaluation of chlorhexidine-impregnated sponges for preventing catheterrelated infections in critically ill adults in the Dressing Study. Crit. Care Med. 40(1), 11-17 (2012).

- This manuscript considers the economic aspects of catheter care, specifically the use of chlorhexidine-soaked sponges at the catheter entry site.

9 Webster J, Gillies D, O'riordan E, Sherriff KL, Rickard CM. Gauze and tape and transparent polyurethane dressings for central venous catheters. Cochrane Database Syst. Rev. (11), CD003827 (2011).

10 Barbour ME, Maddocks SE, Wood NJ, Collins AM. Synthesis, characterization, and efficacy of antimicrobial chlorhexidine hexametaphosphate nanoparticles for applications in biomedical materials and consumer products. Int. J. Nanomedicine 8, 3507-3519 (2013).

- This manuscript is the first report of the chlorhexidine hexametaphosphate material applied in the study described here.

11 Wood NJ, Jenkinson HF, Davis SA, Mann S, O’Sullivan DJ, Barbour ME. Chlorhexidine hexametaphosphate nanoparticles as a novel antimicrobial coating for dental implants. J. Mater. Sci. 26(6), 201 (2015).

12 Bellis CA, Nobbs AH, O'sullivan DJ, Holder JA, Barbour ME. Glass ionomer cements functionalised with a concentrated paste of chlorhexidine hexametaphosphate provides dose-dependent chlorhexidine release over at least 14 months. J. Dent. 45, 53-58 (2016).

- This manuscript illustrates the potential longevity of the antimicrobial function provided by chlorhexidine hexametaphosphate.

13 Miles AA, Misra SS, Irwin JO. The estimation of the bactericidal power of the blood. J. Hyg. (Lond.) 38(6), 732-749 (1938).
14 Cartwright L, Poulsen MS, Nielsen HM et al. In vitro placental model optimization for nanoparticle transport studies. Int. J. Nanomedicine 7, 497-510 (2012).

15 Roper JA, Williamson RC, Bally B et al. Ultrasonic stimulation of mouse skin reverses the healing delays in diabetes and aging by activation of Racl. J. Invest. Dermatol. 135(11), 2842-2851 (2015).

16 Ullman AJ, Cooke ML, Mitchell M et al. Dressings and securement devices for central venous catheters (CVC). Cochrane Database Syst. Rev. 9, CD010367 (2015).

17 Safdar N, O'horo JC, Ghufran A et al. Chlorhexidineimpregnated dressing for prevention of catheter-related bloodstream infection. A meta-analysis*. Crit. Care Med. 42(7), 1703-1713 (2014).

18 Jenks M, Craig J, Green W, Hewitt N, Arber M, Sims A. Tegaderm CHG IV securement dressing for central venous and arterial catheter insertion sites: a NICE medical technology guidance. Appl. Health Econ. Health Policy 14(2), 135-149 (2015).

19 Jeanes A, Bitmead J. Reducing bloodstream infection with a chlorhexidine gel IV dressing. Br. J. Nurs. 24(19), S14-S19 (2015).

20 Jones DS, Lorimer CP, Mccoy CP, Gorman SP. Characterization of the physicochemical, antimicrobial, and drug release properties of thermoresponsive hydrogel copolymers designed for medical device applications. J. Biomed. Mater. Res. B Appl. Biomater. 85(2), 417-426 (2008).

21 Agarwal A, Nelson TB, Kierski PR et al. Polymeric multilayers that localize the release of chlorhexidine from biologic wound dressings. Biomaterials 33(28), 6783-6792 (2012).

22 Lopez-Garcia J, Lehocky M, Humpolicek P, Saha P. HaCaT keratinocytes response on antimicrobial atelocollagen substrates: extent of cytotoxicity, cell viability and proliferation. J. Funct. Biomater. 5(2), 43-57 (2014).

23 John G, Becker J, Schwarz F. Effects of taurolidine and chlorhexidine on SaOS-2 cells and human gingival fibroblasts grown on implant surfaces. Int. J. Oral. Maxillofac Implants 29(3), 728-734 (2014).

24 Shaw TJ, Martin P. Wound repair at a glance. J. Cell Sci. 122, 3209-3213 (2009).

25 Gurtner GC, Werner S, Barrandon Y, Longaker MT. Wound repair and regeneration. Nature 453, 314-321 (2008). 